\title{
Cord Blood Transplantation Following Reduced-Intensity Conditioning for Epstein-Barr Virus-Associated Hemophagocytic Lymphohistiocytosis during Systemic Lupus Erythematosus Treatment
}

\author{
Takuro Kuriyama, Noriaki Kawano, Kiyoshi Yamashita, and Ikuo Kikuchi
}

\begin{abstract}
Epstein-Barr virus-associated hemophagocytic lymphohistiocytosis (EBV-HLH) is a serious disorder in which monoclonal growth of T cells infected with EBV and macrophage activation cause pancytopenia, high fever and acute liver failure. Patients with chemotherapy- or immunosuppression-resistant EBV-HLH require allogeneic hematopoietic stem cell transplantation (allo-HSCT), but patients who have no sibling donors may not have time to wait for an unrelated donor. In pediatric patients, there are some reports on allogeneic cord blood transplantation (allo-CBT) for the treatment of EBV-HLH; however, in adult patients, reports of allo-CBT for EBV-HLH are quite limited. The present case of a 20-year-old woman with chemotherapy-resistant EBV-HLH and systemic lupus erythematosus (SLE) who underwent allo-CBT following reduced-intensity conditioning (RIC-CBT). She achieved and maintained a complete donor type and the EBV-DNA load, and the titers of anti-double stranded DNA and antinuclear antibodies became negative. It is therefore considered that RIC-CBT is an effective treatment option for adult onset HLH. However, because the effectiveness of allo-HSCT for SLE remains unclear and transplant-related mortality is high, it is recommended that allo-HSCT for SLE is restricted to patients concomitant with oncohematological disease as with our present case. [J Clin Exp Hematop 56(2):126-129, 2016〕
\end{abstract}

Keywords: cord blood transplantation, hemophagocytic lymphohistiocytosis, systemic lupus erythematosus

\section{INTRODUCTION}

Hemophagocytic lymphohistiocytosis (HLH) is a syndrome of excessive immune activation characterized by various clinical symptoms of extreme inflammation. The key pathophysiology of this syndrome is hyperactivation of cytotoxic T lymphocytes and macrophages, as well as hypercytokinemia, resulting in hemophagocytosis, pancytopenia and various other symptoms including high fever, acute liver failure, and splenomegaly. ${ }^{1-3}$ HLH is classified into primary HLH and secondary HLH. The primary form of HLH shows clear familial inheritance or genetic causes, such as mutation in PRF1, MUNC13-4 and STX11 genes. ${ }^{4-6}$ Secondary HLH

Received: May 17, 2016

Revised : June 13, 2016

Accepted: June 14, 2016

Department of Internal Medicine, Miyazaki Prefectural Miyazaki Hospital, Miyazaki, Japan

Corresponding author: Dr. Takuro Kuriyama, Department of Internal Medicine, Miyazaki Prefectural Miyazaki Hospital, 5-30 Kitatakamatsu-machi, Miyazaki 8808510, Japan

E-mail: t.kuri@me.com is associated with viral, bacterial, and fungal infections, as well as malignant disorders and rheumatologic disorders. Epstein-Barr virus (EBV) is the most frequent infection associated with HLH, often referred to as virus-associated hemophagocytic syndrome. ${ }^{?}$

EBV-HLH is a serious disorder in which monoclonal growth of $\mathrm{T}$ cells infected with EBV and activated macrophages cause pancytopenia, high fever and acute liver failure because of primary infection or reactivation of EBV. Immunosuppressive therapies including administration of steroids are utilized as the primary treatment of EBV-HLH, thus patients with immunosuppression-resistant EBV-HLH require allogeneic hematopoietic stem cell transplantation (allo-HSCT) therapy. Immunosuppression-resistant EBVHLH is a rapidly advancing disease, thus patients who have no sibling donors often do not have time to wait for an unrelated donor. In pediatric patients, there are some reports of cord blood transplantation (CBT) for EBV-HLH, but in adult patients, reports of allo-CBT for EBV-HLH are limited.

We present here an adult patient who received unrelated umbilical $\mathrm{CBT}$ after a reduced-intensity conditioning regimen (RIC-CBT) for EBV-HLH while undergoing systemic 
lupus erythematosus (SLE) treatment.

\section{CASE REPORT}

A 20-year-old Japanese woman was attending our hospital regularly for SLE and lupus nephritis from the age of 15 years old. She had been treated with steroids, cyclophosphamide and mizoribine, and she received double filtration plasmapheresis once a month and oral tacrolimus from 18 years old. At this point, the SLE disease activity index (SLEDAI) was 8 (proteinuria, low complement and increased DNA binding). ${ }^{8}$ She was referred to our hospital because of high fever lasting for two weeks. Her body temperature was $38.6^{\circ} \mathrm{C}$, her breath and heart sounds were normal and she had no lymphadenopathy. Blood examination revealed a hemoglobin level of $6.9 \mathrm{~g} / \mathrm{dL}$, platelet count of $28 \times 10^{9} / \mathrm{L}$, white blood cell count of $1.22 \times 10^{9} / \mathrm{L}$, and the following blood chemistry results: blood urea nitrogen, $24.9 \mathrm{mg} / \mathrm{dL}$; creatinine, $1.4 \mathrm{mg} / \mathrm{dL}$; total bilirubin, $0.53 \mathrm{mg} / \mathrm{dL}$; aspartate aminotransferase, $16 \mathrm{U} / \mathrm{L}$; alanine aminotransferase, $8 \mathrm{U} / \mathrm{L}$; lactase dehydrogenase, $782 \mathrm{U} / \mathrm{L}$; C-reactive protein, $1.23 \mathrm{mg} / \mathrm{dL}$; ferritin, 1,045 ng/dL; and soluble IL-2 receptor, 22,700 U/ $\mathrm{mL}$. Bone marrow aspiration revealed a hypocellular marrow (nucleated cell count, $36 \times 10^{9}$ cells $/ \mathrm{L}$ ) and spindle-shaped abnormal lymphocytes accounted for $4 \%$ nucleated cells, macrophages $7 \%$ and phagocytes $1.2 \%$ (Fig. $1 \mathrm{a}-1 \mathrm{~d}$ ).
Abnormal cell surface markers were not detected by flow cytometry and chromosomal analysis revealed normal female karyotype. She had a history of infection with EBV; EBVviral capsid antigen immunoglobulin $\mathrm{M}$ antibody was negative, EBV-viral capsid antigen immunoglobulin $\mathrm{G}$ antibody was positive. Measurement of the EBV DNA level in peripheral blood showed an increase to 57,000 copies and Southern blot analysis revealed clonal expansion of EBV-infected cells (Fig. 1e). On the basis of these results, she was diagnosed with EBV-HLH. Furthermore, as it took time for these results to become available, she was treated with CHOP (cyclophosphamide: $750 \mathrm{mg} / \mathrm{m}^{2}$ on day 1 ; vincristine: $1.4 \mathrm{mg} / \mathrm{m}^{2}$ on day 1; doxorubicin: $50 \mathrm{mg} / \mathrm{m}^{2}$ on day 1 ; and prednisolone: 100 mg on days 1-5) because we also considered the possibility of lymphoma-associated hemophagocytic syndrome. Her fever pattern and pancytopenia improved after chemotherapy. The examination outcomes were available before the second round of chemotherapy, and she was therefore treated with CHOP plus etoposide. After a second round of chemotherapy, the EBV DNA level showed a decrease to 440 copies, but it did not become negative and it showed an increase to 24,000 copies after a third round of chemotherapy. We therefore diagnosed the patient with chemotherapy-resistant EBV-HLH and obtained her informed consent for allo-CBT, which she received from HLA-A and DR, double-loci, mismatched unrelated umbilical cord blood because she had no sibling
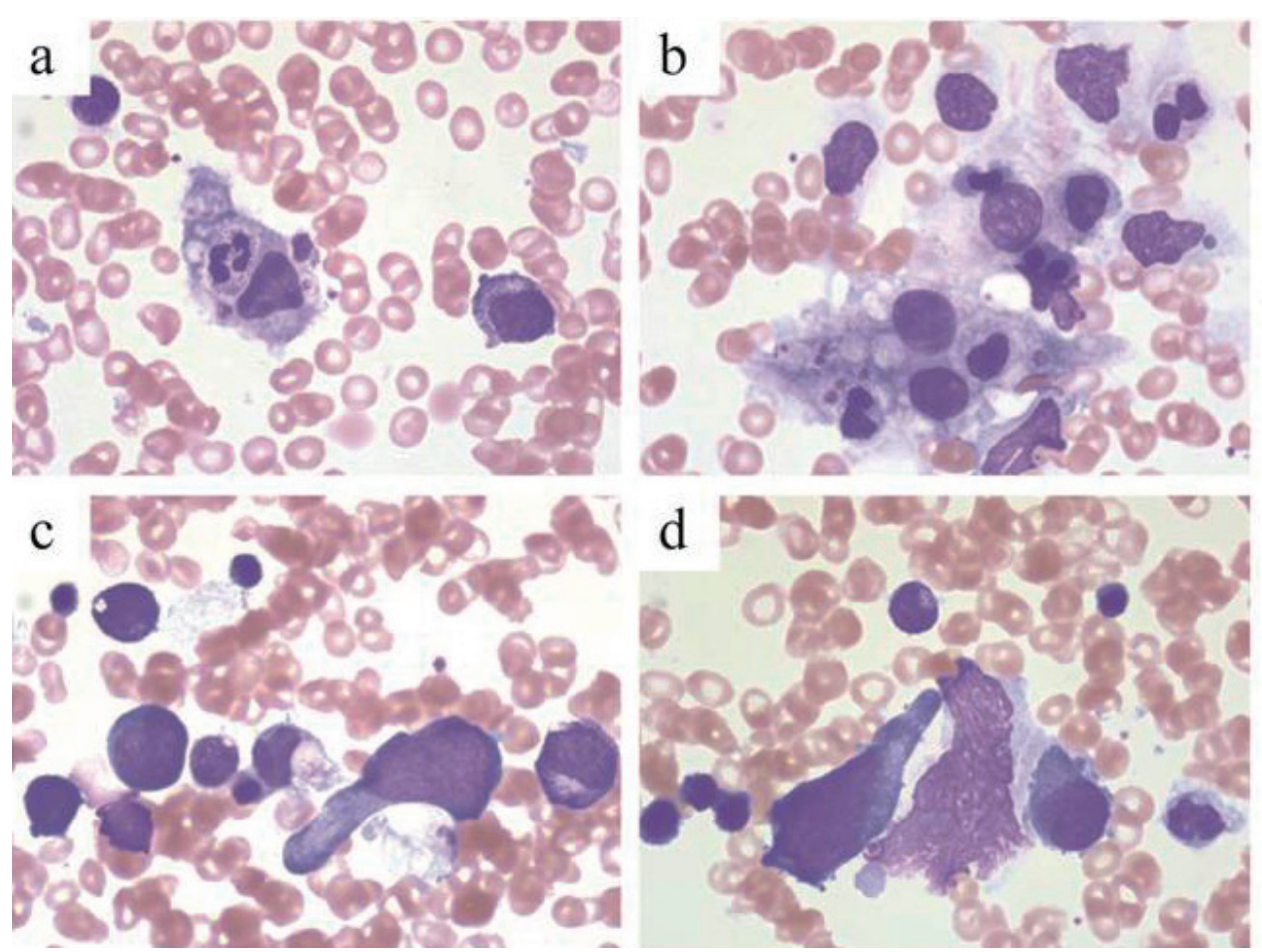

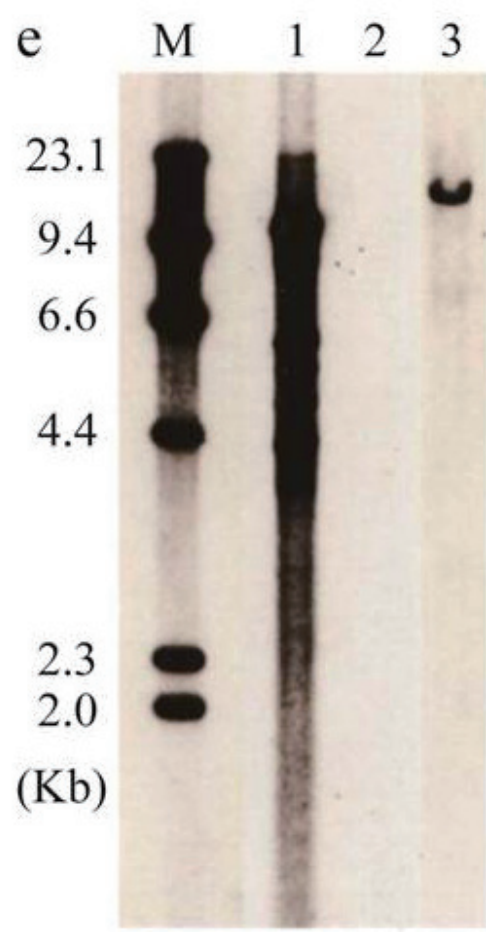

Fig. 1. Bone marrow aspirate smear at onset of hemophagocytosis. (1a, $1 \boldsymbol{b}$ ) Hemophagocytosis in bone marrow. (1c, $\mathbf{1 d})$ Spindle-shaped abnormal lymphocytes in bone marrow. (1e) Southern blot analysis revealed clonal expansion of Epstein-Barr virus-infected cells. M, size marker; 1, positive control; 2, negative control; 3, patient's bone marrow cells 
donor and could not afford to wait for an unrelated donor. She received a conditioning regimen of $25 \mathrm{mg} / \mathrm{m}^{2}$ fludarabine (days -8 to -4 ), $1.25 \mathrm{mg} / \mathrm{kg}$ antithymocyte globulin (ATG; days -7 and -6 ), $40 \mathrm{mg} / \mathrm{m}^{2}$ melphalan (days -3 and -2 ), and 4 Gy of total body irradiation (day -1). Prophylaxis for graftversus-host disease (GVHD) consisted of tacrolimus and mycophenolate mofetil. The donor was male, his blood type was $\mathrm{B}^{+}$(recipient: $\mathrm{A}^{+}$major mismatch), and donor cell level was $2.25 \times 10^{7}$ cells $/ \mathrm{kg}$ and $\mathrm{CD} 34^{+}$cell level was $1.26 \times 10^{5}$ cells $/ \mathrm{kg}$.

The clinical course is shown in Fig. 2. The patient developed a high fever when she received ATG on day -7, but was afebrile the next day, and there were no other adverse events during conditioning chemotherapy. Tacrolimus was given from day -1 , and mycophenolate mofetil was given from day 0 . There were no adverse events $\geq$ grade 3 , other than hematological toxicity and febrile neutropenia until neutrophil engraftment. Engraftment of neutrophils ( $>0.5 \times 10^{9}$ cells/L) was achieved on day 13. Chimerism analysis showed complete donor type on day 21 , and no GVHD $\geq \operatorname{grade} 2$ occurred. The EBV-DNA load was negative on day 28 and it remained negative from then on. She was treated with foscarnet for cytomegalovirus antigenemia from day 30 and renal function elapsed without exacerbation. At the completion of therapy for cytomegalovirus antigenemia, she was discharged from our hospital on day 57.

The titer of anti-double stranded DNA antibody and antinuclear antibody (ANA) were 40 and 1,280 before HSCT; they showed a gradual decline and became negative on day 180. The SLE disease activity index score improved from 8 before HSCT to 4 after HSCT. The creatinine level remained

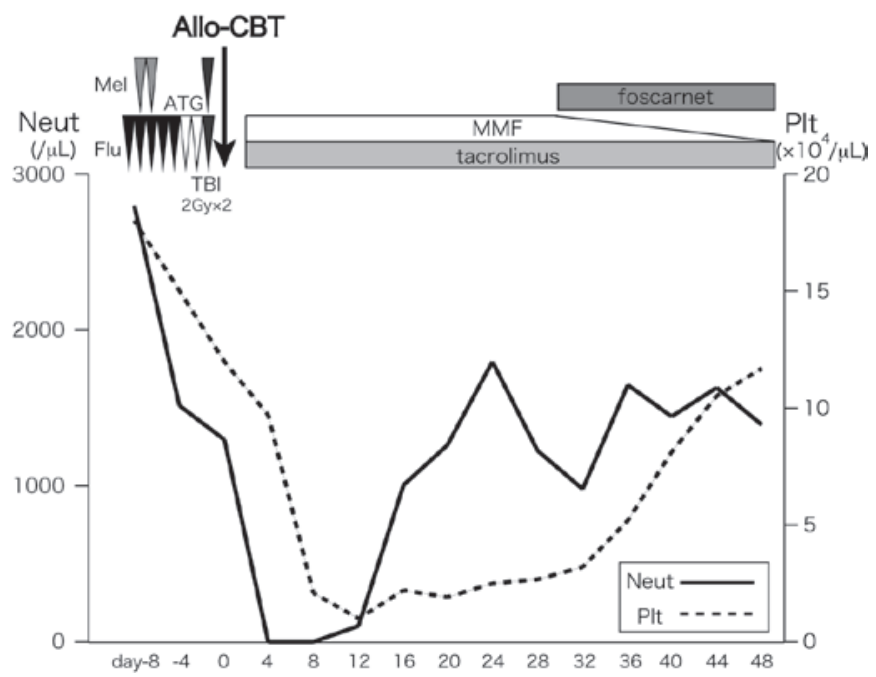

Fig. 2. The course of cord blood transplantation.

ATG, rabbit antithymocyte globulin; Flu, fludarabine, Mel, and melphalan; TBI, total body irradiation; Allo-CBT, allogeneic cord blood transplantation; MMF, mycophenolate mofetil; Neut, neutrophil; Plt, platelet approximately the same before, during, and after HSCT.

\section{DISCUSSION}

Despite the form of HLH, hyperproduction of inflammatory cytokines by activated cytotoxic $\mathrm{T}$ lymphocytes and macrophages plays a central role in the pathogenesis of HLH, and phagocytosis by macrophages of erythrocytes, leukocytes, platelets and their precursors in the bone marrow is a hallmark of HLH. The most important point of HLH treatment is remediation of hypercytokinemia, for which immunosuppressant medication, including steroids and cyclosporine, is used. Etoposide is a key drug for treatment of HLH because it regulates activated macrophages; however, its use in combined immunosuppression and chemotherapy is less certain. Some adult HLH patients were treated according to the HLH-2004 protocol; however, this protocol was primarily developed for pediatric patients. ${ }^{9}$ Thus, the previous reports on the treatment of HLH including EBV infection are almost all pediatric patients and there are only a few reports on treatment of HLH in adult patients.

HSCT is the only possible cure for immunosuppressionresistant $\mathrm{HLH}$, including for EBV-HLH. In pediatric patients with primary HLH, no significant difference in survival was reported between myeloablative conditioning regimen (MAC) and RIC, bone marrow transplantation and CBT. ${ }^{10}$ The immunosuppression-resistant HLH patient's general condition is almost always poor and they may not have time to wait for an unrelated donor, RIC-CBT is therefore a valid option for treatment of this form of HLH. In EBV infection, MAC and RIC have similar event free survival for chronic active EBV infection (CAEBV), although RIC had a better overall survival rate than MAC. ${ }^{11}$ However, these patients had good control for chronic active EBV infection before HSCT, which is not necessarily applicable to immunosuppression-resistant EBV-HLH.

In RIC for HLH, graft failure was induced by recipient's abnormal T cells; therefore, ATG is a key drug for HSCT with the aim of suppression of recipient's T cells. Alemtuzumab had not been approved for use in Japan at that time, the use of ATG was important, not only for GVHD prophylaxis but also suppression of recipient's T cells. The optimal dosage and timing of ATG for HLH were unknown. It was reported that the patients who received alemutuzumab distal to HSCT (day -22 to -19) had a high incidence of GVHD and patients who received proximal to HSCT (day-12 to -9) had a high incidence of developing mixed chimerism. ${ }^{12}$ To minimize the risk of graft failure, it was determined that the optimal date of administration of ATG was day -7 and -6 because the half-life in blood of ATG is about two weeks.

The effectiveness of allogeneic HSCT (allo-HSCT) for standard treatment-resistant autoimmune diseases including SLE is still controversial. Especially, there were few reports 
on allo-HSCT for the treatment of SLE. Marmont et al. reported that patients with SLE complicated by pure red cell aplasia who received allo-HSCT, although it led to remission of pure red cell aplasia, ANA still persisted. ${ }^{13}$ Our case had not only remission of EBV-HLH, but also sustained negative titers of anti-double stranded DNA antibody and ANA. The mechanisms of the effectiveness of allo-HSCT in autoimmune diseases are considered to be immunomodulation by immunosuppressive conditioning, immune-mediated destruction of autoreactive cells and tolerization of peripheral autoreactive/alloreactive $\mathrm{T}$ cells by regulatory T cells. ${ }^{14}$ It was reported that the transplant-related mortality was $22.1 \%$ at 2 years and $30 \%$ at 5 years. ${ }^{15}$ Because the effectiveness of allo-HSCT for SLE is unclear and the transplant-related mortality is high, it recommended that allo-HSCT for SLE is restricted to SLE patients concomitant with oncohematological disease such as our present case. ${ }^{16}$

In conclusion, RIC-CBT led to complete remission of chemotherapy-resistant EBV-HLH during SLE treatment and combined administration of ATG may have prevented graft failure and controlled HLH in the present case. The titer of anti-double stranded DNA antibody and ANA were negative for the duration of the study, but long-term follow-up is needed. We therefore consider that RIC-CBT is an effective treatment option for adult-onset chemotherapy- or immunosuppression-resistant HLH, same as for pediatric HLH, where treatment options are currently limited.

\section{CONFLICTS OF INTEREST}

The authors declare no competing financial interests.

\section{REFERENCES}

1 Filipovich A, McClain K, Grom A: Histiocytic disorders: recent insights into pathophysiology and practical guidelines. Biol Blood Marrow Transplant 16:S82-S89, 2010

2 Janka GE: Familial and acquired hemophagocytic lymphohistiocytosis. Eur J Pediatr 166:95-109, 2007

3 Akashi K, Hayashi S, Gondo H, Mizuno S, Harada M, et al.: Involvement of interferon-g and macrophage colony-stimulating factor in pathogenesis of haemophagocytic lymphohistiocytosis in adults. Br J Haematol 87:243-250, 1994

4 Stepp SE, Dufourcq-Lagelouse R, Le Deist F, Bhawan S, Certain S, et al.: Perforin gene defects in familial hemophagocytic lymphohistiocytosis. Science 286:1957-1959, 1999

5 Feldmann J, Callebaut I, Raposo G, Certain S, Bacq D, et al.: Munc13-4 is essential for cytolytic granules fusion and is mutated in a form of familial hemophagocytic lymphohistiocytosis (FHL3). Cell 115:461-473, 2003
6 Zur Stadt U, Schmidt S, Kasper B, Beutel K, Diler AS, et al.: Linkage of familial hemophagocytic lymphohistiocytosis (FHL) type-4 to chromosome $6 \mathrm{q} 24$ and identification of mutations in syntaxin 11. Hum Mol Genet 14:827-834, 2005

7 Ishii E, Ohga S, Imashuku S, Yasukawa M, Tsuda H, et al.: Nationwide survey of hemophagocytic lymphohistiocytosis in japan. Int J Hematol 86:58-65, 2007

8 Bombardier C, Gladman DD, Urowitz MB, Caron D, Chang $\mathrm{CH}$ : Derivation of the SLEDAI. A disease activity index for lupus patients. The Committee on Prognosis Studies in SLE. Arthritis Rheum 35:630-640, 1992

9 Henter JI, Horne A, Aricó M, Egeler RM, Filipovich AH, et al.: HLH-2004: Diagnostic and therapeutic guidelines for hemophagocytic lymphohistiocytosis. Pediatr Blood Cancer 48:124131, 2007

10 Sawada A, Ohga S, Ishii E, Inoue M, Okada K, et al.: Feasibility of reduced-intensity conditioning followed by unrelated cord blood transplantation for primary hemophagocytic lymphohistiocytosis: A nationwide retrospective analysis in Japan. Int J Hematol 98:223-230, 2013

11 Kawa K, Sawada A, Sato M, Okamura T, Sakata N, et al.: Excellent outcome of allogeneic hematopoietic SCT with reduced-intensity conditioning for the treatment of chronic active EBV infection. Bone Marrow Transplant 46:77-83, 2011

12 Marsh RA, Vaughn G, Kim MO, Li D, Jodele S, et al.: Reduced-intensity conditioning significantly improves survival of patients with hemophagocytic lymphohistiocytosis undergoing allogeneic hematopoietic cell transplantation. Blood 116:5824-5831, 2010

13 Marmont AM, Bacigalupo A, Gualandi F, Bregante S, van Lint MT, et al:: Systemic lupus erythematosus complicated with thymoma and pure red cell aplasia (PCRA). CR of both complications following thymectomy and allogeneic haematopoietic SCT (HSCT), but persistence of antinuclear antibodies (ANA). Bone Marrow Transplant 49:982-983, 2014

14 Sykes M, Nikolic B: Treatment of severe autoimmune disease by stem-cell transplantation. Nature 435:620-627, 2005

15 Daikeler T, Hügle T, Farge D, Andolina M, Gualandi F, et al.: Allogeneic hematopoietic SCT for patients with autoimmune diseases. Bone Marrow Transplant 44:27-33, 2009

16 Marmont du Haut Champ AM: Hematopoietic stem cell transplantation for systemic lupus erythematosus. Clin Dev Immunol 2012:380391, 2012 